\title{
ANÁLISE DA INFLUÊNCIA DA TERAPIA DE CONSCIÊNCIA CORPORAL NA QUALIDADE DE VIDA DE UM GRUPO DE MULHERES MASTECTOMIZADAS.
}

Mariana Romanholi Palma ${ }^{1}$, Fernanda Stellutti Magrini Pachioni ${ }^{2}$, Andressa Carvalho Viscone ${ }^{1}$, Lara Nery Peixoto ${ }^{3}$, Ana Beatriz Salvatori Machado ${ }^{4}$, Larissa Borba André ${ }^{4}$, Thaís Delgado Volpe ${ }^{4}$, Isabela Penteado Novaes ${ }^{4}$, Felipe Novaes Anadão ${ }^{4}$, Edna Maria do Carmo ${ }^{5}$, Cristina Elena Prado Teles Fregonesi ${ }^{6}$.

${ }^{1}$ Discentes do Programa de Pós-Graduação Lato Sensu em Fisioterapia da UNESP - Presidente Prudente, SP. ${ }^{2}$ Discente do Programa de Pós-Graduação Strictu Sensu em Fisioterapia da - UNESP. Presidente Prudente, SP. ${ }^{3}$ Discente do Programa de Pós-graduação Residência em Fisioterapia da - UNESP. Presidente Prudente, SP. ${ }^{4}$ Discentes em Fisioterapia - UNESP. Presidente Prudente, SP. ${ }^{5}$ Docente do Departamento de Fisioterapia da UNESP. Presidente Prudente, SP. ${ }^{6}$ Docente do Departamento de Fisioterapia e do Programa de Pós-Graduação Stricto Sensu em Fisioterapia da UNESP. Presidente Prudente, SP. E-mail: marianaromanholi@hotmail.com

\section{RESUMO}

O câncer de mama gera alto impacto físico e psicológico nas mulheres e acarretam consequências negativas sobre a qualidade de vida da mulher, interferindo na sua imagem e consciência corporal. O objetivo do estudo foi analisar a eficácia da Terapia de Consciência Corporal (TCC), terapias mente-corpo, na qualidade de vida de mulheres submetidas à mastectomia. Participaram 6 mulheres que haviam feito mastectomia. Foram realizadas 16 sessões de TCC, de 50 minutos, 2 vezes por semana. Foi aplicado o questionário de qualidade de vida SF-36, antes e após o tratamento. As escalas avaliadas não apresentaram redução significativa $(p<0,05)$, mas pode-se

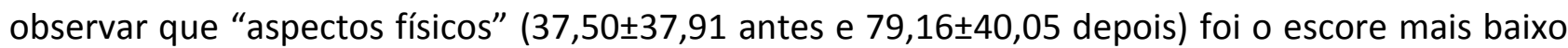
antes das sessões e o de maior alteração. Após a mastectomia, algumas mulheres podem apresentar comprometimentos físicos e psíquicos e é de extrema importância que os tratamentos atuem de forma integral e global.

Palavras-chave: câncer de mama, mastectomia, imagem corporal, qualidade de vida, terapias mente-corpo.

\section{INTRODUÇÃO}

O câncer de mama é a neoplasia mais freqüente entre as mulheres no mundo e é o segundo mais incidênte entre todos os cânceres de acordo com a International Agency for Research on Cancer ${ }^{(1)}$.

Esta patologia tem alto impacto psicológico, pois incide sobre o principal símbolo da feminilidade, sexualidade e maternidade, comprometendo não apenas as condições físicas destas mulheres, como também sua saúde psíquica ${ }^{(2,3)}$. A perda da mama gera sentimentos de rejeição e inferioridade, isso porque a representação do corpo desempenha papel marcante na construção da auto-imagem ${ }^{(4)}$.

Os tratamentos coadjuvantes (quimioterapia, radioterapia e hormonioterapia) têm efeitos colaterais, que levam principalmente à perda do estado funcional e à fadiga crônica. Efeitos estes 
que acarretam consequências negativas sobre a qualidade de vida da mulher de forma geral e interferem na elaboração de sua imagem e consciência corporal ${ }^{(5,2)}$. Portanto, a reabilitação após o procedimento cirúrgico não deve limitar-se apenas a aspectos físicos, visto que a saúde emocional influencia diretamente no quadro clínico geral.

A melhora da consciência corporal tem sido descrita como um elemento-chave ou um mecanismo de ação que proporciona benefícios à saúde. Frequentemente classificadas como terapias corpo-mente, envolvem atenção e o conhecimento das sensações corporais internas. Dentre estas, destacamos a Terapia de Consciência Corporal (TCC), desenvolvida pelo psicanalista

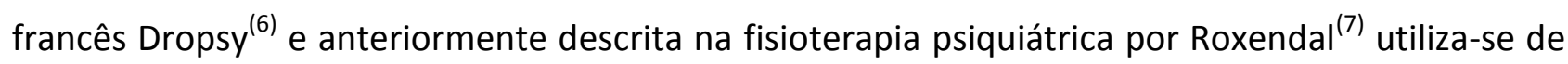
respiração, movimentos e consciência para tentar restaurar o equilíbrio entre corpo e mente. 0 aspecto mais importante da TCC é fazer o paciente recuperar o contato com seu corpo através do movimento e estímulos sensoriais ${ }^{(8)}$.

Muitos estudos demonstram os benefícios dessa terapia em diferentes patologias ${ }^{(8-10)}$, porém não há evidências científicas do uso da TCC em mulheres pós mastectomia.

Diante disto, é fundamental a comprovação científica para a utilização da TCC como alternativa de tratamento com mulheres acometidas pelo câncer de mama, visto que acarretam conseqüências físicas e emocionais desfavoráveis a qualidade de vida da mulher. Portanto, o objetivo do presente estudo foi analisar a influência da TCC na qualidade de vida de mulheres submetidas à mastectomia.

\section{MÉTODOS}

O presente estudo foi realizado no Centro de Estudos e Atendimento em Fisioterapia e Reabilitação (CEAFIR) da Faculdade de Ciências e Tecnologia da Universidade Estadual Paulista (FCT/UNESP).

A população analisada foi composta por 6 pacientes, do gênero feminino, com média de faixa etária de 52,33 $\pm 3,82$ anos, que haviam se submetido à cirurgia de retirada do câncer de mama (mastectomia). Para participação no estudo, as pacientes deveriam ter consentimento médico, ausência de metástase diagnosticada, não poderiam estar em tratamento com quimioterapia ou radioterapia e tempo de pós-operatório entre zero a 10 anos (independente do tipo de cirurgia).

As voluntárias foram devidamente informadas sobre o tipo de tratamento e o objetivo da pesquisa e após concordarem, assinaram um termo de consentimento livre e esclarecido 
permitindo a publicação de seus dados no estudo. Esta pesquisa foi aprovada pelo Comitê de Ética da Universidade Estadual Paulista (FCT-UNESP), (CAAE: 03195912.7.0000.5402).

Foram coletados informações das pacientes, como dados pessoais e tempo de cirurgia. Foi aplicado o questionário de qualidade de vida Study's Short Form-36 (SF-36), traduzido e validado para a Língua Portuguesa por Ciconelli ${ }^{(11)}$. Trata-se de um instrumento composto por 36 itens que avaliam oito escalas: capacidade funcional; aspectos físicos; dor; estado geral de saúde; vitalidade; aspectos sociais; aspectos emocionais e saúde mental. Cada escala pode ter um escore que varia de zero a cem, que corresponde a um pior e melhor estado de saúde, respectivamente.

O programa de terapia de consciência corporal foi constituído por 16 sessões, duas vezes por semana, com duração de 50 minutos. Foi composto por vivências que compreendem movimentos sutis e precisos, que correspondem à exata fisiologia dos músculos, sem forçar sua amplitude, de forma a permitir que o participante conheça melhor seu corpo. Essas vivências foram baseadas em métodos idealizados pelos fisioterapeutas Serge Peyrot, criador da Terapia Morfoanalítica, e Thérèse Bertherat, criadora da Antiginástica.

A condução das vivências de consciência corporal teve o intuito de levar a participante a entrar em contato com as sensações do seu próprio corpo, com enfoque sensorial na pele, na reeducação respiratória, flexibilidade, relaxamento, equilíbrio, coordenação motora, propriocepção e socialização.

As sessões foram conduzidas pausadamente e com todas em silêncio, para que tivessem contato maior com o seu próprio corpo e percebessem cada parte dele e suas inter-relações. Em algumas vivências eram repetidos movimentos várias vezes, de forma lenta, com o objetivo de chamar atenção para o movimento executado. A proposta era de proporcionar uma organização corpórea mais global e equilibrada, para levar à uma organização mais funcional. A condução verbal era seguida dos pés para cabeça ou da cabeça para os pés e depois para os membros superiores.

Nas sessões foram utilizados recursos terapêuticos auxiliares (colchonetes, cunha de espuma, bolinhas de tênis, espaguete de piscina cortados ao meio, bolas de borracha, bolas suíças, disco de propriocepção e de equilíbrio), com objetivo de chamar a atenção para um local preciso, obtendo-se mais percepção da região solicitada.

As sessões eram iniciadas com um momento breve, onde cada participante deveria entrar em contato com seu corpo e tomar a consciência corporal. Elas eram instruídas a realizar movimentos voltados a estimulação da ativação sensorial, focados em uma relação com a 
estabilidade do chão, marcha, posicionamento do tronco e a manutenção do equilíbrio. Ao longo da sessão, era instalada a técnica de respiração diafragmática, da qual todos os movimentos foram ensinados e praticados com padrões respiratórios integrados.

No decorrer de cada sessão os exercícios eram destinados a promover a conscientização da tensão e relaxamento muscular, melhorar a coordenação, descanso e relaxamento consciente. Métodos de relaxamento foram praticados sempre associados a exercícios de respiração.

As sessões eram finalizadas com um momento em grupo, onde cada participante compartilhava as sensações corporais e os sentimentos vividos durante a terapia, de forma à enriquecer o grupo e explorar a percepção corporal, uma vez que o relato das sensações experimentadas por outras pessoas pode chamar atenção para a sua auto-percepção.

Os dados foram apresentados como média \pm desvio padrão. A análise do questionário SF36 foi realizada por meio do teste T de Student, com nível de significância de 5\%.

\section{RESULTADOS}

O tempo de pós operatório da amostra analisada variou de 2 à 10 anos (Tabela 1).

Tabela 1. Tempo de pós-operatório. $n=6$.

\begin{tabular}{cc}
\hline Tempo de cirurgia & Sujeitos \\
\hline 2 anos & 1 \\
$3-5$ anos & 3 \\
6-8 anos & 1 \\
$9-10$ anos & 1 \\
\hline
\end{tabular}

As oito áreas avaliadas do questionário SF-36 estão representadas na tabela 2. 
Tabela 2 - Valores médios e desvio padrão de cada área analisada do questionário de qualidade de vida (SF- 36) antes e após 16 sessões, seguida por p-valor.

$n=6$.

\begin{tabular}{lccc} 
Área & Inicial & Após 16 sessões & p-valor \\
\hline & & & \\
CF & $59,16 \pm 24,57$ & $64,16 \pm 29,73$ & 0,547 \\
AF & $37,50 \pm 37,91$ & $79,16 \pm 40,05$ & 0,054 \\
DOR & $46,83 \pm 25,67$ & $54,16 \pm 19,50$ & 0,457 \\
EGS & $68,33 \pm 25,67$ & $78,33 \pm 14,30$ & 0,349 \\
VIT & $57,5 \pm 23,16$ & $56,66 \pm 28,22$ & 0,129 \\
AS & $57,5 \pm 41,68$ & $64,58 \pm 39,06$ & 0,518 \\
AE & $61,10 \pm 28,84$ & $66,66 \pm 51,63$ & 0,363 \\
SM & $60,00 \pm 24,84$ & $60,66 \pm 33,12$ & 0,899 \\
$\mathrm{p}<0,05$ & & &
\end{tabular}

CF- capacidade funcional; AF- aspectos físicos; EGS- estado geral de saúde; VIT- vitalidade; AS- aspectos sociais; AEaspectos emocionais; SM- saúde mental.

\section{DISCUSSÃO}

Embora as escalas avaliadas não tenham apresentado redução significativa $(p<0,05)$, nota-se através das médias que "aspectos físicos" foi o escore mais baixo antes das sessões, mostrando que o maior impacto na qualidade de vida das pacientes analisadas foi dado pela limitação física, já que todas as outras escalas, exceto dor, também apresentaram média inicial maior que 55 pontos (considerado boa qualidade de vida).

Um estudo realizado em um centro de atenção à saúde da mulher, com 110 mulheres que tiveram câncer de mama, analisou a qualidade de vida das mesmas através de aplicações de questionários e observaram que os melhores escores foram obtidos no domínio físico e os piores no domínio psicológico. Embora nenhum escore tenha atingido valor menor que 55 pontos, sabese que a doença afeta de forma geral a qualidade de vida e que esses resultados podem ser o reflexo da assistência multiprofissional oferecida por este centro de atenção a saúde ${ }^{(12)}$. Isso reforça que é necessário um atendimento mais amplo à essas mulheres não focando apenas nos comprometimentos físicos como também nos psicossociais.

Outro estudo com 42 mulheres que terminaram tratamento oncológico devido ao câncer de mama, evidenciou alterações na capacidade funcional que levam à prejuízos na realização de suas atividades cotidianas e na participação social. Essas mulheres referiram que a dependência e a perda da identidade geram angústias e medos, isso prejudica a função emocional e a qualidade 
de vida das mesmas, já que o câncer de mama tem importante influência nos aspectos emocionais da mulher, devido as mudanças em sua independência e autonomia, além de incapacidades e da idealizada ou real proximidade com a morte, que levam aos medos, angústias e sentimento de discriminação ${ }^{(13)}$.

As mulheres analisadas neste estudo apresentaram maior comprometimento físico, os escores "saúde mental" (60,00 $\pm 24,84$ antes; 60,66 $\pm 33,12$ depois) e "aspectos emocionais" $(61,10 \pm 28,84$ antes; $66,66 \pm 51,63$ depois) já se apresentaram maior que 55 pontos antes do inicio das sessões e pouco se alteraram. Nota-se que o fator psíquico não foi tão acometido na fase em que analisamos. Isto pode se justificar pelo fato da ausência de metástase ter sido um fator de exclusão da pesquisa e da maioria das pacientes ter tempo de pós operatório maior que 3 anos. No entanto, nosso enfoque foi o tratamento corpo-mente, para englobar todos os aspectos que se enquadram na qualidade de vida.

Melhorar a qualidade de vida após o câncer de mama pode ser um desafio para os profissionais da área da saúde, já que diz respeito a percepção subjetiva do indivíduo sobre sua saúde em geral. Portanto, é necessário não adotar uma postura reducionista, mas sim tratar o paciente como um todo, afinal a saúde é definida como bem estar físico, mental e social.

A terapia de consciência corporal mostrou melhoria nos fatores mais alterados das mulheres analisadas (aspectos físicos e dores) e visa tratar o paciente de forma global, levando o mesmo a entrar em contato com as sensações de seu próprio.

\section{CONCLUSÃO}

Após a mastectomia, algumas mulheres podem apresentar comprometimentos físicos e psíquicos que alteram sua qualidade de vida. Com isto, é de extrema importância que os tratamentos não sejam focados apenas no maior comprometimento e sim que as terapias visem à promoção de saúde dessas mulheres. A TCC pode ser considerada como opção. No entanto, é importante que outros estudos com TCC em mastectomizadas sejam realizados com maior número de sessões e amostra.

\section{REFERÊNCIAS}

1. International Agency for Research on Cancer. http://www.iarc.fr/en/cancertopics/index.php. [homepage on the Internet]. 
2. Ahlin J, Abreu MA, Vilarinho R, Madureira F. Analise da influencia da pratica do ciclismo indoor na composicao corporal de mulheres que tiveram cancer de mama .Revista Mackenzie de Educacao Fisica e Esporte. 2007;6 (3): 67-72.

3. Menezes NNT, Schultz VL, Peres RS. Impacto psicológico do diagnóstico do câncer de mama: um estudo a partir dos relatos de pacientes em um grupo de apoio Estudos de Psicologia. 2012; 17(2): 233-40. http://dx.doi.org/10.1590/S1413-294X2012000200006

4. Alves PC, Silva APS, Santos MCL, Fernandes AFC. Conhecimento e expectativas de mulheres no pré-operatório da mastectomia. Rev Esc Enferm USP. 2010; 44(4):989-95. http://dx.doi.org/10.1590/S0080-62342010000400019

5. Santos DB, Vieira EM. Imagem corporal de mulheres com câncer de mama: uma revisão sistemática da literatura. Ciência \& Saúde Coletiva. 2011; 16 (5):2511-22. http://dx.doi.org/10.1590/S1413-81232011000500021

6. Dropsy J. Leva i sin kropp. Stockholm: Aldus, 1975.

7. Roxendal G. Body awareness therapy and the body awareness scale. Gothenburg: Dept. Rehabilitation Medicine, University of Gothenburg; 1985.

8. Gard G. Body awareness therapy for patients with fibromyalgia and chronic pain. Disabil Rehabil. 2005; 27:725-28. http://dx.doi.org/10.1080/09638280400009071

9. Gyllestein AL, Ekdahl, C, Hansson L. Long-term effectiveness of Basic Body Awareness Therapy in psychiatric outpatient care. A randomized controlled study. European Journal of Physiotherapy. 2009. 11(1): 2-12.

10. Catalan-Matamoros D, Helvik-Skjaerven L, Labajos-Manzanares MT, Martı́nez-de-SalazarArboleas A, Sa'nchez-Guerrero E. A pilot study on the effect of Basic Body Awareness Therapy in patients with eating disorders: a randomized controlled Trial. Clinical Rehabilitation. 2011.25(7) 617-26.

11. Ciconelli RM, Ferraz MB, Santos W, Meinão I, Quaresma MR. Tradução para a língua portuguesa e validação do questionário genérico de avaliação de qualidade de vida SF-36 (Brasil SF-36). Rev Bras Reumatol. 1999; 39(3): p 143-52.

12. Huguet PR, Morais SS, Osis MJD, Pinto-Neto AM, Gurgel MSC. Qualidade de vida e sexualidade de mulheres tratadas de câncer de mama. Rev Bras Ginecol Obstet. 2009; 31(2):61-7. http://dx.doi.org/10.1590/S0100-72032009000200003

13. Vendrusculo-Fangel LM, Panobianco MS, Kebbe LM, Almeida AM, Gozzo TO. Qualidade de vida e desempenho de atividades cotidianas após tratamento das neoplasias mamárias. Acta Paul Enferm. 2013; 26(1):93-100. http://dx.doi.org/10.1590/S0103-21002013000100015 